\title{
Lipid complexes of amphotericin B: the competitive picture
}

Invasive fungal infections are among. the most important causes of morbidity and mortality in immunosuppressed patients, such as cancer patients treated with intensive chemotherapy, solid organ transplant recipients and AIDS patients. As the overall prognosis for patients with invasive fungal infections remains poor, there is a crucial need to improve the treatment of these infections. Since the 1950s, the standard agent prescribed for systemic antifungal therapy has been amphotericin B. Its broad spectrum of antifungal activity makes it an attractive agent for the empirical treatment of patients who may be infected with yeasts or moulds. Its track record as the primary therapy for most invasive fungal infections is well established, and it is still widely regarded as the agent of choice for serious, life threatening infections even though its use is fraught with toxicity problems. Nephrotoxicity is the most severe problem and is often dose-limiting. Given the importance of systemic mycoses in clinical medicine, the design of new, less toxic preparations with enhanced therapeutic effectiveness has accelerated.

During the last decade alternative formulations of amphotericin B based on the use of particulate drug delivery systems such as liposomes or other lipid particles have been described $[1,2]$. The promising results of animal and mode of action studies along with technological advances in lipid-complex production methodology have led to their clinical application.

Prospective comparative trials are difficult to conduct because of the complex nature of the patients' underlying diseases and the frequent use of empirical treatment for presumptive infections. In the absence of published prospective comparative studies, therefore, it is not possible to compare directly the different formulations. However, it is important to review the characteristics of these agents and draw some conclusions on their clinical effectiveness and side-effect profile.

The most well-established of the lipid-complexed formulations of amphotericin B is AmBisome (NeXstar Pharmaceutical Inc.), which is now widely available. This is the only true liposomal formulation. It is being used increasingly in Europe for the treatment of serious, life-threatening fungal infection and is the most widely investigated lipid-based formulation of amphotericin B. A New Drug Application for AmBisome has been submitted for approval to the United States Food and Drug Administration. The application seeks approval for AmBisome as primary treatment for confirmed and presumptive fungal infection, and for the treatment of fungal infections refractory to conventional amphotericin B treatment. The use of AmBisome clinically is supported by extensive published data [3]. Over 150 publications on the safety and efficacy of AmBisome illustrate how beneficial this agent has been. Data include its use in confirmed and suspected infections in adults and children, including those with neutropenia; in patients undergoing transplantation or haemofiltration; and for the empirical treatment of fever of unknown origin. To date, AmBisome has been used to treat $>10000$ patients worldwide at the recommended dose of 1 $3 \mathrm{mg} / \mathrm{kg}$ daily and, in some patients, to over $10 \mathrm{mg} / \mathrm{kg}$ daily. AmBisome is clinically effective throughout the dose range. Representative studies show up to $77 \%$ clinical efficacy at a daily dose of $2.4 \mathrm{mg} / \mathrm{kg}$ in candidosis and aspergillosis [3]. In cryptococcosis, a $79 \%$ response rate at a mean daily dose of $2.8 \mathrm{mg} / \mathrm{kg}$ has been reported [4]. High doses, up to $5 \mathrm{mg} / \mathrm{kg}$ daily, have been used successfully in clinical trials to treat patients with proven or highly suspected aspergillus infections in whom therapy with conventional amphotericin B had failed. What is not entirely clear is whether or not dose escalation or higher doses achieves greater clinical response. At the high doses, the reported incidence of adverse effects appears to be $<5 \%$. Of note is the resolution of infection in patients with prolonged neutropenia.

AmBisome appears to be effective (with a response rate up to $80 \%$ ) and well-tolerated in the treatment of invasive fungal infections in immunocompromised patients. It is a significantly safer alternative to conventional amphotericin B in children and adults. The clinical and mycological efficacy of both conventional amphotericin B and AmBisome are equivalent on a dose-to-dose comparison in adult and paediatric patients. When used as salvage therapy in patients who had prior treatment with conventional amphotericine $\mathrm{B}$, the response rate has been $40-50 \%$ for infections with Aspergillus and Candida spp. [3]. Furthermore, AmBisome appears to have an underlying immunomodulating effect by enhancing effector cell function against fungal cells [1]. The studies on 
the accumulation of AmBisome around fungal lesions and its mode of action at the fungal cell membrane are well accepted.

Another commercially available formulation of amphotericin B is Amphocil (amphotericin B colloidal dispersion; Sequus Pharmaceuticals Inc.). This is a non-liposomal amphotericin B-lipid complex. Amphocil was approved for marketing in the UK in 1994 and subsequently in several other countries. The tolerance and efficacy of this formulation have been assessed in two clinical trials $[5,6]$ and independent studies. However, the clinical data are still too limited to allow firm conclusions to be drawn. Nevertheless, the collective data on compassionate use of Amphocil in patients with invasive fungal infection is very encouraging. The optimal dosage and duration of use of Amphocil has not been established. The manufacturer recommends starting at a dosage of $1 \mathrm{mg} / \mathrm{kg}$ as a single infusion given over a $60-90-\mathrm{min}$ period with a step-wise increase to $3-4 \mathrm{mg}$ as required. If a patient experiences acute reactions (in addition to fever, chills and rigors, there is continued concern regarding anaphylactoid reactions) or cannot tolerate the infusion volume within $90 \mathrm{~min}$, the infusion time should be extended.

If Amphocil is to achieve more widespread use, a consensus of the most appropriate dosage indications for maximum clinical efficacy and agreement on toxicity tolerances need to established on the basis of published and unpublished studies.

The third Amphotericin B formulation under review is Abelcet (amphotericin B lipid complex; The Liposome Company Inc.). This is another non-liposomal amphotericin B-lipid complex. Abelcet received marketing approval in the UK in 1995 and has also been approved for sale in the USA, Spain, Luxemburg, Iceland and Scandinavia. It is indicated for the treatment of severe invasive candidosis and is also indicated as second-line treatment of invasive aspergillosis, cryptococcosis in HIV patients and miscellaneous fungal infections. The drug appears to be cleared rapidly from the serum. High tissue levels have been recorded in animals, especially in the lung and liver, followed by rapid clearance of the drug from the tissue area over $24 \mathrm{~h}$ [7]. Human data regarding accumulation of Abelcet in lung tissue appears to be based on post-mortem tissue from one patient [8]. The benefit of rapid clearance from the circulation is not very clear, as a number of fungal pathogens causing acute pulmonary disease, including Aspergillus, cause vasculitis by penetrating the walls of the vessels into the lumen. Large fungal masses are found in these vessels. It is here that therapeutic levels of amphotericin B are required. Furthermore, very little is known about the precise mode of action of Abelcet at the cellular level. The initial clinical trials were conducted in the USA. The size and quality of these studies varied considerably and the conclusions that can be drawn are limited in the absence of confirmatory studies. Examination of animal studies, summary data in abstract form, and a limited number of publications $[9,10]$ are the basis of recommendations for patients. The recommended dosage for severe infections in human patients is $5 \mathrm{mg} / \mathrm{kg}$ daily given as a single infusion for at least 14 days. Responses to treatment times are highly variable. As with the other lipid-based formulations, the key advantage of Abelcet is significantly reduced toxicity, although it appears that a reduced dose of $2.5 \mathrm{mg} / \mathrm{kg}$ daily may be necessary to reduce acute side-effects.

The cost of the lipid-associated formulations is a matter of concern. Predicting the cost of treatment is by its very nature speculative. Consideration has to be made of the underlying medical condition of the patient as well as concomitant therapies. The cost of treating adverse effects should also be considered. Comparative cost analyses suggest that the cost of treating confirmed fungal infections with AmBisome, Amphocil or Abelcet is not significantly different. Ultimately, the cost will depend on the duration of treatment and the dosage used. Furthermore, many patients are treated empirically in the absence of definitive laboratory tests.

The three lipid-associated formulations of amphotericin B appear to be far better tolerated than conventional amphotericin B, enabling higher doses of the drug to be given with less risk of harmful side-effects. Although the results of open trials with these formulations have been encouraging, proof of their improved effectiveness will come only from comparative trials. These trials will have to be well designed with firm grounds for diagnosis (mycological and serological) and clear endpoints for response. In the meantime, it is reasonable to use a lipid-associated formulation of amphotericin B in a patient with an invasive fungal infection who has renal failure or in whom there have been serious renal or other side-effects with the conventional formulation. The choice of amphotericin B lipid formulation can be made only after careful consideration of the evidence-based scientific literature and clinical trial data and should not be influenced by marketing and advertising strategies.

M. D. RICHARDSON Regional Mycology Reference Laboratory, West Glasgow Hospitals University NHS Trust and University of Glasgow, Glasgow G11 6NU.

\section{References}

1. Abu-Salah KM. Amphotericin B: an update. Br J Biomed Sci 1996; 53: $122-133$.

2. Brajtburg I, Bolard J. Carrier effects on biological activity of amphotericin B. Clin Microbiol Rev 1996; 9: 512-531.

3. Ng TTC, Denning DW. Liposomal amphotericin B (AmBi- 
some) therapy in invasive fungal infections. Evaluation of United Kingdom compassionate use data. Arch Intern Med 1995; 155: 1093-1098.

4. Coker RJ, Viviani M, Gazzard GB et al. Treatment of cryptococcosis with liposomal amphotericin B (AmBisome) in 23 patients with AIDS. AIDS 1993; 7: 829-835.

5. Oppenheim BA, Herbrecht R, Kusne S. The safety and efficacy of amphotericin B colloidal dispersion in the treatment of invasive mycoses. Clin Infect Dis 1995; 21: 1145-1153.

6. Bowden RA, Cays M, Gooley T, Mamelok RD, van Burik JA Phase 1 study of amphotericin B colloidal dispersion for the treatment of invasive fungal infections after marrow transplant. $J$ Infect Dis 1996; 173: 1208-1215.
7. Olsen SJ, Swerdel MR, Blue B, Clark JM, Bonner DP. Tissue distribution of amphotericin B lipid complex in laboratory animals. J Pharm Pharmacol 1991; 43: 831-835.

8. Abelcet Product Monograph. The Liposomal Company Ltd. 1995.

9. Oravcova E, Mistrik M, Sakalova A et al. Amphotericin B lipid complex to treat invasive fungal infections in cancer patients: report of efficacy and safety in 20 patients. Chemotherapv 1995; 41: 473-476.

10. Sharkey PK, Graybill JR, Johnson ES et al. Amphotericin B lipid complex compared with amphotericin B in the treatment of cryptococcal meningitis in patients with AIDS. Clin Infect Dis 1996; 22: 315-321. 